\title{
A PROPOSED WATTMETER USING MULTIELECTRODE TUBES*
}

\author{
By \\ John R. Pierce \\ (California Institute of Technology, Pasadena, California)
}

$\mathbb{F}$

YOR direct measurement of small amounts of power, or for measurement of power over a wide range of frequencies, dynamometer wattmeters are unsatisfactory, and vacuum tube wattmeters are often resorted to.

Hitherto vacuum tube wattmeters have depended for their operation on the use of tubes having a square-law characteristic, connected in a push-pull circuit so as to avoid indications caused by current or voltage alone. Such a device was described by H. M. Turner and F. T. MacNamara in $1930^{1}$ and was patented by E. Peterson in $1926 .^{2}$ References to this type of wattmeter may also be found in standard works on radio-frequency measurement. ${ }^{3,4}$

An attempt to use a multielectrode tube with coplanar grids for the measurement of power was made by T. B. Wagner. ${ }^{5}$ While the device developed was satisfactory as a voltmeter or ammeter, it must be discounted as a wattmeter, for it is clearly brought out in Wagner's article that readings may be obtained by applying voltage or current alone, a condition of zero power. Moreover, it is stated that the output of the tube used is proportional to the sum of the effects of voltage and current if applied separately.

The author wishes to propose a new method of measuring power through the use of multielectrode tubes.

It may be shown of some multielectrode tubes that under certain conditions the application of alternating voltage to two controlling elements or grids results in a change in the direct-current component of the plate current proportional to the product of the voltages at the grids and the cosine of their phase angle. Hence such tubes may be used in the construction of a vacuum tube wattmeter.

* Decimal classification: R240. Original manuscript received by the Institute, September 30, 1935; revised manuscript received by the Institute, January 2, 1936.

${ }^{1}$ H. M. Turner and F. T. MacNamara, Proc. I.R.E., vol. 18, pp. 17431747; October, (1930). 1926.

${ }_{2}^{2}$ Patent No. 1,586,553, E. Peterson, Bell Telephone Laboratories, June,

${ }^{3}$ Terman, "Measurements in Radio Engineering," pp. 31-32.

${ }^{4}$ Hund, "High-Frequency Measurements," pp. 302-303.

5 T. B. Wagner, Elec. Eng., vol. 53, pp. 1621-1623; December, (1934). 
Of the tubes investigated by the author, the mixer type of tube, such as the type $2 \mathrm{~A} 7$ or $6 \mathrm{~A} 7$, seemed best adapted for use as a wattmeter tube. The disposition of electrodes in a type $2 \mathrm{~A} 7$ or $6 \mathrm{~A} 7$ tube is shown in Fig. 1. $G_{2}$ and $G_{3}$ are held positive at a fixed potential above that of the cathode, and $G_{1}$ and $G_{4}$ are biased to be negative with respect to the cathode. Alternating-current potentials proportional to voltage and current are then applied to $G_{1}$ and $G_{4}$.

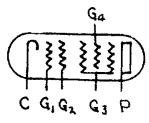

Fig. 1

In such a case, the current passing through $G_{1}$ is dependent only on the potential of $G_{1}$, being independent of the potential of $G_{4}$. The

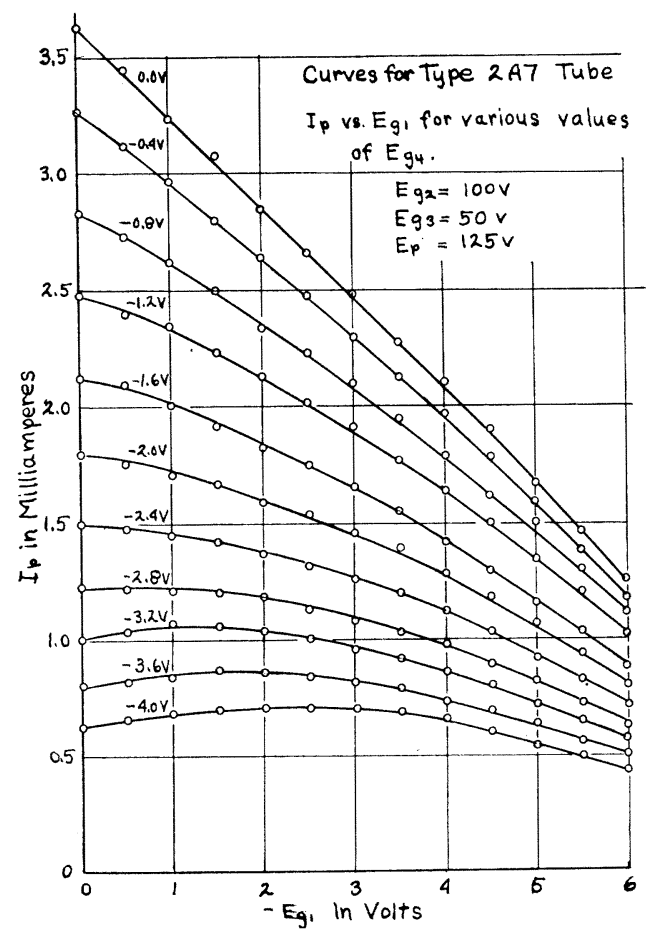

Fig. 2

proportion of this current reaching the plate is, however, dependent only on the potential of $G_{4}$. Thus if the characteristics are linear for both $G_{1}$ and $G_{4}$, we may expect that the plate current will be represented in the form 


$$
I_{p}=A E_{G 1}+B E_{G 1} E_{G 4}+C E_{G 4}+D
$$

where $A, B, C$, and $D$ are constants.

The first and third terms are necessarily of a purely alternatingcurrent nature and would not register on a direct-current meter in the plate circuit. The direct-current component of the product term is proportional to the power, since $E_{G 1}$ and $E_{G 4}$ are proportional to alternating voltage and current.

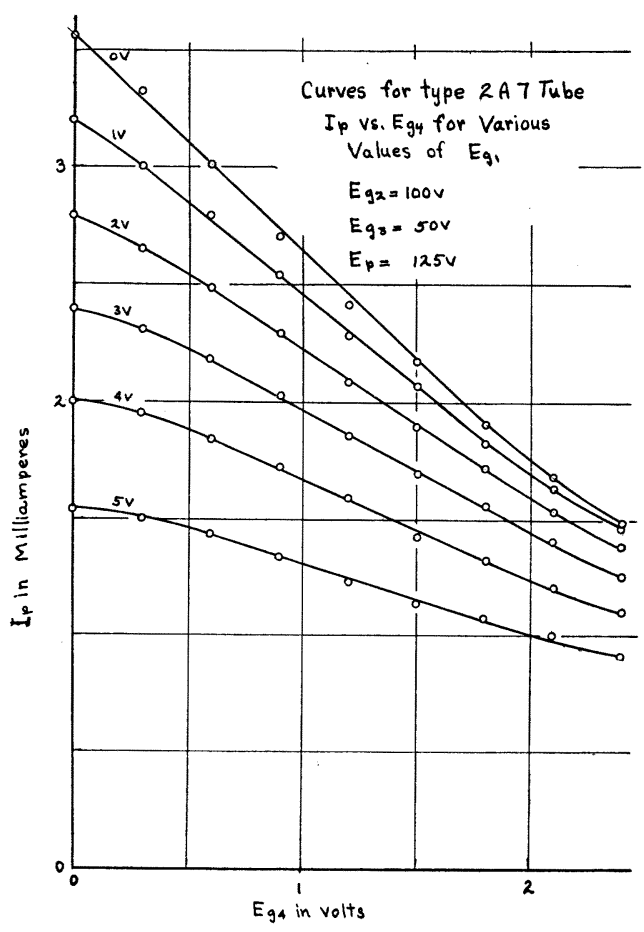

Fig. 3

The actual static characteristics, experimentally obtained by the author, for a 2A7 tube are shown in Figs. 2 and 3. Fig. 2 is a plot of $I_{p}$ vs. $E_{G 1}$ for various values of $E_{G 4}$, and Fig. 3 is a plot of $I_{p}$ vs. $E_{G 4}$ for various values of $E_{G 1}$.

If a region can be located such that within it $I_{p}$ vs. $E_{G 1}$ for various values of $E_{G 4}$ can be represented by a family of straight lines which, extended, pass through a common point, and if in this region $I_{p}$ vs. $E_{G 4}$ is, for some value of $E_{G 1}$, a straight line, then the plate current of the tube can be represented by (1) and the tube may be used as a wattmeter tube. 
Figs. 2 and 3 should, if sufficiently accurate, enable us to find such a region if it existed. Unfortunately, there is no large region of linear variation of $I_{p}$ with $E_{G 1}$, although something approximating such a region may be found. Further, curves such as these can hardly be accurate enough to give final evidence of the degree of linearity, and can best be used to show under what operating conditions linearity may be expected.

Variation of the tube characteristic from linearity is best detected by applying various alternating-current potentials to one grid alone and noting the variation in the direct-current component of the plate current due to "rectification." Making such tests, the author found that

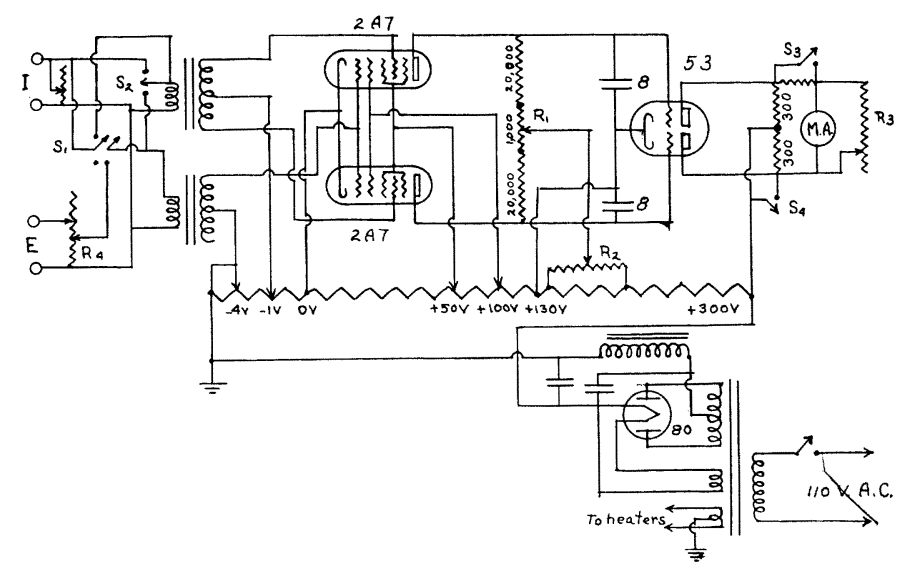

Fig. 4

the variation in the direct-current component of the plate current caused by an alternating voltage applied to $G_{1}$ alone was great enough to preclude the use of a single tube in a wattmeter, since "rectification" would necessarily result in readings due to current or voltage alone.

Linearity is much more closely approached in the variation of $I_{p}$ with $E_{G 4}$ alone. The best result obtained was a change in the directcurrent component of $I_{p}$ caused by the application of an alternating voltage to $G_{4}$ alone not more than three per cent of that for the same alternating voltage applied to both $G_{1}$ and $G_{4}$.

It is possible that tubes similar to the type $2 \mathrm{~A} 7$ but having more nearly linear characteristics could be developed. This presents an interesting field of investigation which was closed to the author because of lack of equipment.

In view of the nonlinearity of the characteristics of the tubes available, a push-pull type of circuit was adopted to check experimentally 
the operation of a wattmeter constructed on the principles outlined above. The circuit is shown in Fig. 4. The apparatus was built in a selfcontained form with variable shunts and voltage dividers to cover a range from 0.01 to 1.00 amperes and 1 to 200 volts, and with switches to allow for the use of the instrument as a square-law voltmeter or ammeter as well as a wattmeter. A direct-current amplifier was provided to allow the use of a rugged one-milliampere meter in place of the microammeter which would otherwise have been required.

The instrument was tested at fifty cycles. The curves shown in Figs. 5 and 6 demonstrate that at this frequency the instrument behaves as a wattmeter should. Fig. 5 is a plot of deflection as a watt-

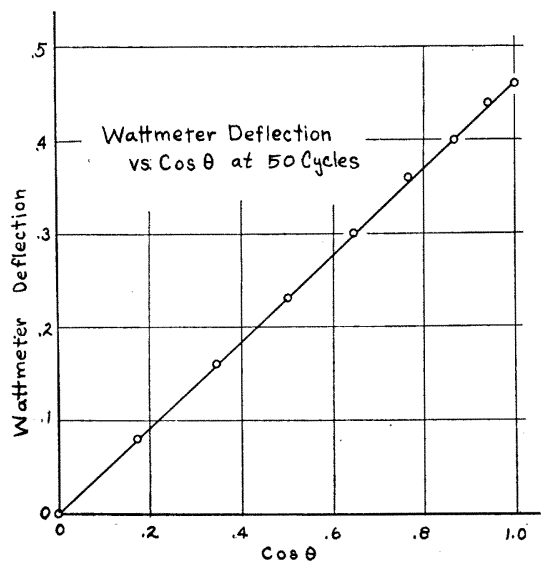

Fig. 5

meter with constant voltage and current vs. cosine of the phase angle, and shows that the instrument behaves correctly as far as power factor is concerned. In obtaining this experimental plot the author used an iron-core phase shifting transformer, such as is used in the calibration of watt-hour meters. Fig. 6 is a plot of indicated amperes (the square root of scale reading) for the instrument as an ammeter vs. actual amperes as measured. by an ammeter. This illustrates that the deflection is actually proportional to the product of the voltages at the grids, in this case each proportional to the current.

With this circuit, the frequency limitation is of course that of the transformers. Obviously, the phase shift of the transformers does not matter as long as the transformers in the voltage and current sides have equal phase shifts, as they will if they are identical. It is important, however, that the ratio of transformation does not vary with frequency and that the transformers do not in any way distort the wave 
form. In the present state of the art there should be small difficulty in obtaining transformers having a substantially constant ratio of transformation over the audio range, and giving no appreciable distortion of wave form. With such transformers the device would function as satisfactorily over the entire audio range as it does at fifty cycles.

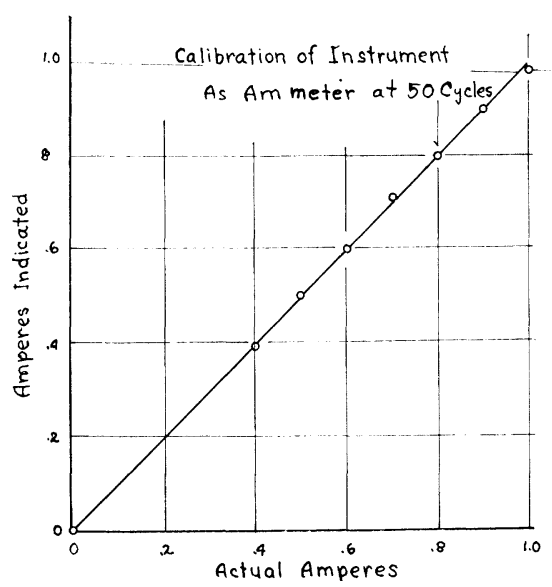

Fig. 6

It should be noted that such an instrument in conjunction with an audio-frequency oscillator would serve admirably as an harmonic analyzer of a zero-beat type, such as described by C. G. Suits. ${ }^{6}$

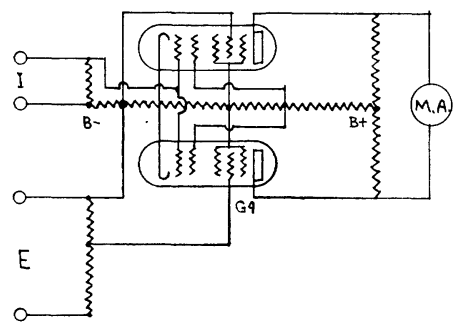

Fig. 7

In extending the range of the wattmeter to cover radio frequencies, transformers must, of course, be avoided. As has been explained, a single tube could be used were its characteristics for both control grids sufficiently linear. Provided the characteristic of one grid only is substantially linear, as in the case of the type $2 \mathrm{~A} 7$ tube, the circuit suggested in Fig. 7 should provide a means of reasonably accurate power

${ }^{6}$ C. G. Suits, Proc. I.R.E., vol. 18, pp. 178-192; January, (1930). 
measurement without the use of transformers. This circuit is symmetrical with respect to the applied current; hence deflections due to current alone are avoided. It is not symmetrical with respect to the applied voltage, but sufficient linearity of the characteristic for $G_{4}$ to avoid immoderate deflections caused by voltage alone is presumed. A certain limitation of this circuit, that one side of the current input and one side of the voltage input must be at the same radio-frequency potential, must be common to all vacuum tube wattmeters not making use of transformers. Direct-current isolation may be obtained through the use of condensers.

In conclusion, it may be asserted that present multielectrode tubes of the mixer type can be utilized to advantage as wattmeter tubes, and that with similar tubes having more nearly linear characteristics, the art of power measurement at low levels and varying frequencies might be considerably advanced. 